\title{
On Certain Hypergeometric Summation Theorems Motivated by the Works of Ramanujan, Chudnovsky and Borwein
}

\author{
M. I. Qureshi \\ Department of Applied Sciences and Humanities, Faculty of Engineering and Technology \\ Jamia Millia Islamia (A central university), Jamia Nagar, New Delhi-110025, INDIA \\ E-mail: miqureshi_delhi@yahoo.co.in \\ Izharul H. Khan \\ Department of Applied Sciences and Humanities, Faculty of Engineering and Technology \\ Jamia Millia Islamia (A central university), Jamia Nagar, New Delhi-110025, INDIA \\ E-mail: izhargkp@rediffmail.com \\ M. P. Chaudhary (Corresponding author) \\ Department of Applied Sciences and Humanities, Faculty of Engineering and Technology \\ Jamia Millia Islamia (A central university), Jamia Nagar, New Delhi-110025, INDIA \\ E-mail: mpchaudhary_2000@yahoo.com
}

\begin{abstract}
In the present paper, we obtain numerical values for Gaussian hypergeometric summation theorems by giving particular values to the parameters $a, b$ and the argument $x$; three summation theorems for ${ }_{2} F_{3}\left(\frac{1}{4}, \frac{3}{4} ; \frac{1}{2}, \frac{1}{2}, 1 ; x\right)$, three summation theorems for ${ }_{4} F_{3}\left(\frac{1}{2}, \frac{1}{2}, \frac{1}{2}, \frac{a+b}{b} ; 1,1, \frac{a}{b} ; x\right)$, two summation theorems for ${ }_{4} F_{3}\left(\frac{1}{2}, \frac{1}{3}, \frac{2}{3}, \frac{a+b}{b} ; 1,1, \frac{a}{b} ; x\right)$, four summation theorems for ${ }_{4} F_{3}\left(\frac{1}{2}, \frac{1}{6}, \frac{5}{6}, \frac{a+b}{b} ; 1,1, \frac{a}{b} ; x\right)$ and ten summation theorems for ${ }_{4} F_{3}\left(\frac{1}{2}, \frac{1}{4}, \frac{3}{4}, \frac{a+b}{b} ; 1,1, \frac{a}{b} ; x\right)$.
\end{abstract}

A.M.S.(M.O.S.) Subject Classification (1991): 33-Special Functions.

Keywords: Ramanujan integrals, Ramanujan series, Ordinary Bessel function of first kind of order $n$, Borwein and Chudnovsky series

\section{Ordinary Bessel Function of First Kind of Order $n$}

$$
J_{n}(x)=\frac{(x / 2)^{n}}{\Gamma(n+1)}{ }_{0} F_{1}\left[\begin{array}{l}
-; \\
n+1 ;
\end{array}\right]
$$

\subsection{Lemma}

If $a, p$ and $n$ are suitably adjusted real or complex numbers such that associated Pochhammer's symbols are welldefined, then we have

$$
(a+p n)=\frac{a\left(\frac{a+p}{p}\right)_{n}}{\left(\frac{a}{p}\right)_{n}}
$$

B. C. Berndt ( pp.352-354) listed all of Ramanujan's series for $\frac{1}{\pi}$ found in the 133 unorganized pages of the second and third notebooks of Ramanujan (1984).

1.2 Seventeen Ramanujan's Series [Berndt, B. C(Part-IV); Hardy, G. H.; Ramanujan, S., 1914; Venkatachala, B. J., 2000]

$$
\begin{aligned}
\mathrm{R}_{4} & \equiv \frac{4}{\pi}=1+\frac{7}{4}\left(\frac{1}{2}\right)^{3}+\frac{13}{4^{2}}\left(\frac{1.3}{2.4}\right)^{3}+\frac{19}{4^{3}}\left(\frac{1.3 .5}{2.4 .6}\right)^{3}+\cdots \\
& =\sum_{n=0}^{\infty} \frac{(6 n+1)\left(\frac{1}{2}\right)_{n}^{3}}{4^{n}(n !)^{3}}
\end{aligned}
$$




$$
\begin{aligned}
& \mathrm{R}_{5} \equiv \frac{16}{\pi}=5+\frac{47}{64}\left(\frac{1}{2}\right)^{3}+\frac{89}{64^{2}}\left(\frac{1.3}{2.4}\right)^{3}+\frac{131}{64^{3}}\left(\frac{1.3 .5}{2.4 .6}\right)^{3}+\cdots \\
& =\sum_{n=0}^{\infty} \frac{(42 n+5)\left(\frac{1}{2}\right)_{n}^{3}}{64^{n}(n !)^{3}} \\
& \mathrm{R}_{6} \equiv \frac{32}{\pi}=(5 \sqrt{5}-1)+\left(\frac{47 \sqrt{5}+29}{64}\right)\left(\frac{1}{2}\right)^{3}\left(\frac{\sqrt{5}-1}{2}\right)^{8}+ \\
& +\left(\frac{89 \sqrt{5}+59}{64^{2}}\right)\left(\frac{1.3}{2.4}\right)^{3}\left(\frac{\sqrt{5}-1}{2}\right)^{16}+\cdots \\
& =\sum_{n=0}^{\infty} \frac{(42 \sqrt{5} n+5 \sqrt{5}+30 n-1)\left(\frac{1}{2}\right)_{n}^{3}}{64^{n}(n !)^{3}}\left(\frac{\sqrt{5}-1}{2}\right)^{8 n} \\
& \mathrm{R}_{7} \equiv \frac{27}{4 \pi}=2+17 \frac{1}{2} \frac{1}{3} \frac{2}{3}\left(\frac{2}{27}\right)+32 \frac{1.3}{2.4} \frac{1.4}{3.6} \frac{2.5}{3.6}\left(\frac{2}{27}\right)^{2}+\cdots \\
& =\sum_{n=0}^{\infty} \frac{(15 n+2)\left(\frac{1}{2}\right)_{n}\left(\frac{1}{3}\right)_{n}\left(\frac{2}{3}\right)_{n}}{(n !)^{3}}\left(\frac{2}{27}\right)^{n} \\
& \mathrm{R}_{8} \equiv \frac{15 \sqrt{3}}{2 \pi}=4+37 \frac{1}{2} \frac{1}{3} \frac{2}{3}\left(\frac{4}{125}\right)+70 \frac{1.3}{2.4} \frac{1.4}{3.6} \frac{2.5}{3.6}\left(\frac{4}{125}\right)^{2}+\cdots \\
& =\sum_{n=0}^{\infty} \frac{(33 n+4)\left(\frac{1}{2}\right)_{n}\left(\frac{1}{3}\right)_{n}\left(\frac{2}{3}\right)_{n}}{(n !)^{3}}\left(\frac{4}{125}\right)^{n} \\
& \mathrm{R}_{9} \equiv \frac{5 \sqrt{5}}{2 \pi \sqrt{3}}=1+12 \frac{1}{2} \frac{1}{6} \frac{5}{6}\left(\frac{4}{125}\right)+23 \frac{1.3}{2.4} \frac{1.7}{6.12} \frac{5.11}{6.12}\left(\frac{4}{125}\right)^{2}+\cdots \\
& =\sum_{n=0}^{\infty} \frac{(11 n+1)\left(\frac{1}{2}\right)_{n}\left(\frac{1}{6}\right)_{n}\left(\frac{5}{6}\right)_{n}}{(n !)^{3}}\left(\frac{4}{125}\right)^{n} \\
& \mathrm{R}_{10} \equiv \frac{85 \sqrt{85}}{18 \pi \sqrt{3}}=8+141 \frac{1}{2} \frac{1}{6} \frac{5}{6}\left(\frac{4}{85}\right)^{3}+274 \frac{1.3}{2.4} \frac{1.7}{6.12} \frac{5.11}{6.12}\left(\frac{4}{85}\right)^{6}+\cdots \\
& =\sum_{n=0}^{\infty} \frac{(133 n+8)\left(\frac{1}{2}\right)_{n}\left(\frac{1}{6}\right)_{n}\left(\frac{5}{6}\right)_{n}}{(n !)^{3}}\left(\frac{4}{85}\right)^{n} \\
& \mathrm{R}_{11} \equiv \frac{4}{\pi}=\frac{3}{2}-\frac{23}{2^{3}} \frac{1}{2} \frac{1.3}{4^{2}}+\frac{43}{2^{5}} \frac{1.3}{2.4} \frac{1.3 .5 .7}{4^{2} .8^{2}}-\cdots \\
& =\sum_{n=0}^{\infty} \frac{(-1)^{n}(20 n+3)\left(\frac{1}{2}\right)_{n}\left(\frac{1}{4}\right)_{n}\left(\frac{3}{4}\right)_{n}}{(n !)^{3} 2^{2 n+1}} \\
& \mathrm{R}_{12} \equiv \frac{4}{\pi \sqrt{3}}=\frac{3}{4}-\frac{31}{3.4^{3}} \frac{1}{2} \frac{1.3}{4^{2}}+\frac{59}{3^{2} .4^{5}} \frac{1.3}{2.4} \frac{1.3 .5 .7}{4^{2} .8^{2}}-\cdots \\
& =\sum_{n=0}^{\infty} \frac{(-1)^{n}(28 n+3)\left(\frac{1}{2}\right)_{n}\left(\frac{1}{4}\right)_{n}\left(\frac{3}{4}\right)_{n}}{(n !)^{3} 3^{n} 4^{n+1}} \\
& \mathrm{R}_{13} \equiv \frac{4}{\pi}=\frac{23}{18}-\frac{283}{18^{3}} \frac{1}{2} \frac{1.3}{4^{2}}+\frac{543}{18^{5}} \frac{1.3}{2.4} \frac{1.3 .5 .7}{4^{2} .8^{2}}-\cdots
\end{aligned}
$$




$$
\begin{aligned}
& =\sum_{n=0}^{\infty} \frac{(-1)^{n}(260 n+23)\left(\frac{1}{2}\right)_{n}\left(\frac{1}{4}\right)_{n}\left(\frac{3}{4}\right)_{n}}{(n !)^{3}(18)^{2 n+1}} \\
& \mathrm{R}_{14} \equiv \frac{4}{\pi \sqrt{5}}=\frac{41}{72}-\frac{685}{5.72^{3}} \frac{1}{2} \frac{1.3}{4^{2}}+\frac{1329}{5^{2} .72^{5}} \frac{1.3}{2.4} \frac{1.3 .5 .7}{4^{2} .8^{2}}-\cdots \\
& =\sum_{n=0}^{\infty} \frac{(-1)^{n}(644 n+41)\left(\frac{1}{2}\right)_{n}\left(\frac{1}{4}\right)_{n}\left(\frac{3}{4}\right)_{n}}{(n !)^{3} 5^{n}(72)^{2 n+1}} \\
& \mathrm{R}_{15} \equiv \frac{4}{\pi}=\frac{1123}{882}-\frac{22583}{882^{3}} \frac{1}{2} \frac{1.3}{4^{2}}+\frac{44043}{882^{5}} \frac{1.3}{2.4} \frac{1.3 .5 .7}{4^{2} .8^{2}}-\cdots \\
& =\sum_{n=0}^{\infty} \frac{(-1)^{n}(21460 n+1123)\left(\frac{1}{2}\right)_{n}\left(\frac{1}{4}\right)_{n}\left(\frac{3}{4}\right)_{n}}{(n !)^{3}(882)^{2 n+1}} \\
& \mathrm{R}_{16} \equiv \frac{2 \sqrt{3}}{\pi}=1+\frac{9}{9} \frac{1}{2} \frac{1.3}{4^{2}}+\frac{17}{9^{2}} \frac{1.3}{2.4} \frac{1.3 .5 .7}{4^{2} \cdot 8^{2}}+\cdots \\
& =\sum_{n=0}^{\infty} \frac{(8 n+1)\left(\frac{1}{2}\right)_{n}\left(\frac{1}{4}\right)_{n}\left(\frac{3}{4}\right)_{n}}{(n !)^{3} 9^{n}} \\
& \mathrm{R}_{17} \equiv \frac{1}{2 \pi \sqrt{2}}=\frac{1}{9}+\frac{11}{9^{3}} \frac{1}{2} \frac{1.3}{4^{2}}+\frac{21}{9^{5}} \frac{1.3}{2.4} \frac{1.3 .5 .7}{4^{2} \cdot 8^{2}}+\cdots \\
& =\sum_{n=0}^{\infty} \frac{(10 n+1)\left(\frac{1}{2}\right)_{n}\left(\frac{1}{4}\right)_{n}\left(\frac{3}{4}\right)_{n}}{(n !)^{3} 9^{2 n+1}} \\
& \mathrm{R}_{18} \equiv \frac{1}{3 \pi \sqrt{3}}=\frac{3}{49}+\frac{43}{49^{3}} \frac{1}{2} \frac{1.3}{4^{2}}+\frac{83}{49^{5}} \frac{1.3}{2.4} \frac{1.3 \cdot 5.7}{4^{2} \cdot 8^{2}}+\cdots \\
& =\sum_{n=0}^{\infty} \frac{(40 n+3)\left(\frac{1}{2}\right)_{n}\left(\frac{1}{4}\right)_{n}\left(\frac{3}{4}\right)_{n}}{(n !)^{3}(49)^{2 n+1}} \\
& \mathrm{R}_{19} \equiv \frac{2}{\pi \sqrt{11}}=\frac{19}{99}+\frac{299}{99^{3}} \frac{1}{2} \frac{1.3}{4^{2}}+\frac{579}{99^{5}} \frac{1.3}{2.4} \frac{1.3 .5 .7}{4^{2} .8^{2}}+\cdots \\
& =\sum_{n=0}^{\infty} \frac{(280 n+19)\left(\frac{1}{2}\right)_{n}\left(\frac{1}{4}\right)_{n}\left(\frac{3}{4}\right)_{n}}{(n !)^{3}(99)^{2 n+1}} \\
& \mathrm{R}_{20} \equiv \frac{1}{2 \pi \sqrt{2}}=\frac{1103}{99^{2}}+\frac{27493}{99^{6}} \frac{1}{2} \frac{1.3}{4^{2}}+\frac{53883}{99^{10}} \frac{1.3}{2.4} \frac{1.3 .5 .7}{4^{2} .8^{2}}+\cdots \\
& =\sum_{n=0}^{\infty} \frac{(26390 n+1103)\left(\frac{1}{2}\right)_{n}\left(\frac{1}{4}\right)_{n}\left(\frac{3}{4}\right)_{n}}{(n !)^{3}(99)^{4 n+2}}
\end{aligned}
$$

The first three series representations $\mathrm{R}_{4}, \mathrm{R}_{5}$ and $\mathrm{R}_{6}$ for $\frac{1}{\pi}$ are found in Ramanujan's papers [Hardy, G. H., 2000, pp.36-37; Ramanujan, S., 1914]. The remaining fourteen series representations $R_{7}, R_{8}, \cdots, R_{20}$ are stated without proof in Ramanujan's papers [Hardy, G. H., 2000, pp.37-38; Ramanujan, S., 1914].

Ramanujan has used the equality sign for his approximations to $\pi$ but he states explicitly that the formulae are correct only to a certain number of decimal places. The symbol $\approx$ had not come into vogue by then.

1.3 Borwein Series [Borwein, J. M., 1987; 1988; 1993]

In 1987, Borwein and Borwein [Borwein, J. M., 1987; Berndt, B. C., 2003, p.193], two computer scientists used a following version of Ramanujan's formula $\mathrm{R}_{20}$ to calculate $\pi$ to 17 million places and found that the formula converges on 
the exact value with far greater efficiency than any previous method. This success proved that Ramanujan's insight was correct.

$$
\begin{gathered}
\mathrm{R}_{21} \equiv \frac{1}{\pi}=12 \sum_{n=0}^{\infty} \frac{(-1)^{n}(6 n) !}{(n !)^{3}(3 n) !} \times \\
\times \frac{[212175710912 \sqrt{61}+1657145277365+n(13773980892672 \sqrt{61}+107578229802750)]}{\{5280(236674+30303 \sqrt{61})\}^{\left(3 n+\frac{3}{2}\right)}}
\end{gathered}
$$

1.4 Chudnovsky Series (Chudnovsky, D. V., 1988)

In 1989, David and Gregory Chudnovsky computed 1011196961 digits of $\pi$, using the series [Berndt, B. C., 2003, p.201]

$$
\mathrm{R}_{22} \equiv \frac{1}{\pi}=12 \sum_{n=0}^{\infty} \frac{(-1)^{n}(6 n) !(13591409+n 545140134)}{(n !)^{3}(3 n) !\left\{(640320)^{3}\right\}^{\left(n+\frac{1}{2}\right)}}
$$

which is similar to those found by Ramanujan.

In 1991, the Chudnovskys computed in excess of 2.16 billion digits. Many details of their work on a largely home-built computer are given in the delightful profile "The Mountains of Pi" (Preston, R., 1992).

The Ramanujan series from $\mathrm{R}_{4}$ to $\mathrm{R}_{20}$ were not proved until 1987, when J. M. and P. B. Borwein proved them in their book [Borwein, J. M, 1987, pp.177-187]. In three further papers (Borwein, J. M, 1987), (Borwein, J. M, 1988) and (Borwein, J. M, 1993), they established several additional formulas of this type. D. V. and G. V. Chudnovsky (1988) not only also proved formulas of this sort, but they, moreover, found representations for other transcendental constants, some involving gamma functions, by hypergeometric series of the same kin.

It should be remarked that Ramanujan has no notation for hypergeometric series [Berndt, B. C (Part-II), p.8]. All formulas are stated by writing out the first few terms in each series.

\section{Main Summation Theorems}

$$
\begin{aligned}
& { }_{2} F_{3}\left[\begin{array}{r}
\frac{1}{4}, \frac{3}{4} ; \frac{-\pi^{2}}{4} \\
\frac{1}{2}, \frac{1}{2}, 1 ;
\end{array}\right]=0 \\
& \left.{ }_{2} F_{3}\left[\begin{array}{r}
\frac{1}{4}, \frac{3}{4} ; \\
\frac{1}{2}, \frac{1}{2}, 1 ;
\end{array}\right]=-\pi^{2}\right]=-J_{0}(\pi)
\end{aligned}
$$

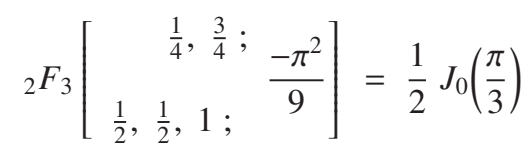

$$
\begin{aligned}
& { }_{4} F_{3}\left[\begin{array}{rr}
\frac{1}{2}, \frac{1}{2}, \frac{1}{2}, \frac{7}{6} ; & \frac{1}{4} \\
1,1, \frac{1}{6} ; & {[}
\end{array}\right]=\frac{4}{\pi} \\
& \left.{ }_{4} F_{3}\left[\begin{array}{c}
\frac{1}{2}, \frac{1}{2}, \frac{1}{2}, \frac{47}{42} ; \\
1,1, \frac{5}{42} ;
\end{array}\right]=\frac{1}{64}\right]=\frac{16}{5 \pi} \\
& \left.{ }_{4} F_{3}\left[\begin{array}{c}
\frac{1}{2}, \frac{1}{2}, \frac{1}{2}, \frac{(375-8 \sqrt{5})}{330} ; \\
1,1, \frac{(45-8 \sqrt{5})}{330} ;
\end{array}\right]=\frac{(47-21 \sqrt{5})}{128}\right]=\frac{(8+40 \sqrt{5})}{31 \pi} \\
& { }_{4} F_{3}\left[\begin{array}{c}
\frac{1}{2}, \frac{1}{3}, \frac{2}{3}, \frac{17}{15} ; \\
1,1, \frac{2}{15} ;
\end{array}\right]=\frac{27}{8 \pi}
\end{aligned}
$$


The equation (28) is known hypergeometric form of Berndt and Rankin [Berndt, B. C, 2003, p.298(3.4)].

$$
\begin{aligned}
& \left.{ }_{4} F_{3}\left[\begin{array}{c}
\frac{1}{2}, \frac{1}{3}, \frac{2}{3}, \frac{37}{33} ; \\
1,1, \frac{4}{33} ;
\end{array}\right]=\frac{4}{125}\right]=\frac{15 \sqrt{3}}{8 \pi} \\
& \left.{ }_{4} F_{3}\left[\begin{array}{r}
\frac{1}{2}, \frac{1}{6}, \frac{5}{6}, \frac{12}{11} ; \\
1,1, \frac{1}{11} ;
\end{array}\right]=\frac{4}{125}\right]=\frac{5 \sqrt{15}}{6 \pi} \\
& \left.{ }_{4} F_{3}\left[\begin{array}{c}
\frac{1}{2}, \frac{1}{6}, \frac{5}{6}, \frac{141}{133} ; \\
1,1, \frac{8}{133} ;
\end{array}\right]=\frac{4}{85}\right]=\frac{85 \sqrt{255}}{432 \pi} \\
& { }_{4} F_{3}\left[\begin{array}{c}
\frac{1}{2}, \frac{1}{4}, \frac{3}{4}, \frac{23}{20} ; \\
1,1, \frac{3}{20} ;
\end{array}\right]=\frac{-1}{3 \pi} \\
& { }_{4} F_{3}\left[\begin{array}{c}
\frac{1}{2}, \frac{1}{4}, \frac{3}{4}, \frac{31}{28} ; \\
1,1, \frac{-1}{28} ;
\end{array}\right]=\frac{16 \sqrt{3}}{9 \pi} \\
& \left.{ }_{4} F_{3}\left[\begin{array}{c}
\frac{1}{2}, \frac{1}{4}, \frac{3}{4}, \frac{283}{260} ; \\
1,1, \frac{23}{260} ;
\end{array}\right]=\frac{-1}{324}\right]=\frac{72}{23 \pi} \\
& \left.{ }_{4} F_{3}\left[\begin{array}{c}
\frac{1}{2}, \frac{1}{4}, \frac{3}{4}, \frac{685}{644} ; \\
1,1, \frac{41}{644} ;
\end{array}\right]=\frac{-1}{25920}\right]=\frac{288 \sqrt{5}}{205 \pi} \\
& \left.{ }_{4} F_{3}\left[\begin{array}{c}
\frac{1}{2}, \frac{1}{4}, \frac{3}{4}, \frac{22583}{21460} ; \\
1,1, \frac{1123}{21460} ;
\end{array}\right]=\frac{-1}{777924}\right]=\frac{3528}{1123 \pi} \\
& { }_{4} F_{3}\left[\begin{array}{c}
\frac{1}{2}, \frac{1}{4}, \frac{3}{4}, \frac{9}{8} ; \\
1,1, \frac{1}{8} ;
\end{array}\right]=\frac{2 \sqrt{3}}{\pi} \\
& { }_{4} F_{3}\left[\begin{array}{c}
\frac{1}{2}, \frac{1}{4}, \frac{3}{4}, \frac{11}{10} ; \\
1,1, \frac{1}{10} ;
\end{array}\right]=\frac{9 \sqrt{2}}{4 \pi} \\
& \left.{ }_{4} F_{3}\left[\begin{array}{c}
\frac{1}{2}, \frac{1}{4}, \frac{3}{4}, \frac{43}{40} ; \\
1,1, \frac{3}{40} ;
\end{array}\right]=\frac{1}{2401}\right]=\frac{49 \sqrt{3}}{27 \pi} \\
& { }_{4} F_{3}\left[\begin{array}{ccc}
\frac{1}{2}, & \frac{1}{4}, \frac{3}{4}, \frac{299}{280} ; & 1 \\
1,1, \frac{19}{280} ; & 9801
\end{array}\right]=\frac{198 \sqrt{11}}{209 \pi} \\
& \left.{ }_{4} F_{3}\left[\begin{array}{c}
\frac{1}{2}, \frac{1}{4}, \frac{3}{4}, \frac{27493}{26390} ; \\
1,1, \frac{1103}{26390} ;
\end{array}\right]=\frac{1}{96059601}\right]=\frac{9801 \sqrt{2}}{4412 \pi}
\end{aligned}
$$


The equation (41) is the correct form of erroneous hypergeometric form of Berndt and Rankin[2003, p.298(3.5)].

The series (28) and (41) are given in sections 13 and 14 of (Ramanujan, S., 1914) and [Ramanujan, S., 1984, p.378].

$$
\begin{gathered}
{ }_{4} F_{3}\left[\begin{array}{r}
\frac{1}{6}, \frac{1}{2}, \frac{5}{6}, \frac{13986156603584 \sqrt{61}+109235375080115}{13773980892672 \sqrt{61}+107578229802750} ; \\
1,1, \frac{212175710912 \sqrt{61}+1657145277365}{13773980892672 \sqrt{61}+107578229802750} ;\{5280(236674+30303 \sqrt{61})\}^{3}
\end{array}\right] \\
=\frac{\{5280(236674+30303 \sqrt{61})\}^{\frac{3}{2}}}{12(212175710912 \sqrt{61}+1657145277365) \pi} \\
{ }_{4} F_{3}\left[\begin{array}{c}
\frac{1}{6}, \frac{1}{2}, \frac{5}{6}, \frac{558731543}{545140134} ; \\
\left.1,1, \frac{13591409}{545140134} ; \frac{-1728}{(640320)^{3}}\right]=\frac{(640320)^{\frac{3}{2}}}{12(13591409) \pi}
\end{array}\right.
\end{gathered}
$$

Above summation theorems for ${ }_{4} F_{3}$ are not available in the mostly available literature of special functions including the monumental works of Prudnikov et. al. [Prudnikov, A. P., 1990, pp.552-563]. The summation theorems presented by equations (42) and (43) are the hypergeometric forms of the Borwein series (20) and Chudnovsky series (21) respectively.

\section{Proof of (22) to (43)}

The hypergeometric summation theorems from (22) to (43) can be established from the results $R_{1}$ to $R_{22}$ respectively.

Proof of (24) : To prove (24), we shall consider the Ramanujan's integral $R_{3}$.

$$
\int_{0}^{\frac{\pi}{2}} \cos \left(\frac{2 \pi \sin ^{2} \theta}{3}\right) d \theta=\frac{1}{2} \int_{0}^{\frac{\pi}{2}} \cos \left(\frac{\pi \sin \theta}{3}\right) d \theta
$$

Using Maclaurin's series for $\cos x=\sum_{n=0}^{\infty} \frac{(-1)^{n} x^{2 n}}{(2 n) !}$, we get

$$
\int_{0}^{\frac{\pi}{2}} \sum_{n=0}^{\infty} \frac{(-1)^{n}\left(\frac{2 \pi \sin ^{2} \theta}{3}\right)^{2 n}}{(2 n) !} d \theta=\frac{1}{2} \int_{0}^{\frac{\pi}{2}} \sum_{n=0}^{\infty} \frac{(-1)^{n}\left(\frac{\pi \sin \theta}{3}\right)^{2 n}}{(2 n) !} d \theta
$$

Now interchanging the order of summation and integration, we get

On simplifying, we get

$$
\begin{gathered}
\sum_{n=0}^{\infty} \frac{(-1)^{n}\left(\frac{4 \pi^{2}}{9}\right)^{n}}{(2 n) !} \int_{0}^{\frac{\pi}{2}} \sin ^{4 n} \theta d \theta=\frac{1}{2} \sum_{n=0}^{\infty} \frac{(-1)^{n}\left(\frac{\pi^{2}}{9}\right)^{n}}{(2 n) !} \int_{0}^{\frac{\pi}{2}} \sin ^{2 n} \theta d \theta \\
\sum_{n=0}^{\infty} \frac{(-1)^{n}\left(\frac{4 \pi^{2}}{9}\right)^{n} \Gamma\left(\frac{4 n+1}{2}\right) \Gamma\left(\frac{1}{2}\right)}{(2 n) ! 2 \Gamma\left(\frac{4 n+2}{2}\right)}=\frac{1}{2} \sum_{n=0}^{\infty} \frac{(-1)^{n}\left(\frac{\pi^{2}}{9}\right)^{n} \Gamma\left(\frac{2 n+1}{2}\right) \Gamma\left(\frac{1}{2}\right)}{(2 n) ! 2 \Gamma\left(\frac{2 n+2}{2}\right)}
\end{gathered}
$$

$$
\begin{gathered}
\sum_{n=0}^{\infty} \frac{\left(\frac{1}{4}\right)_{n}\left(\frac{3}{4}\right)_{n}\left(-\frac{\pi^{2}}{9}\right)^{n}}{\left(\frac{1}{2}\right)_{n}\left(\frac{1}{2}\right)_{n}(1)_{n} n !}=\frac{1}{2} \sum_{n=0}^{\infty} \frac{\left(-\frac{\pi^{2}}{36}\right)^{n}}{(1)_{n} n !} \\
{ }_{2} F_{3}\left[\begin{array}{c}
\left.\frac{1}{4}, \frac{3}{4} ; \frac{-\pi^{2}}{9}\right]=\frac{1}{2}{ }_{0} F_{1}\left[\begin{array}{c}
-; \\
1 ;
\end{array}\right] \\
\frac{1}{2}, \frac{-\pi^{2}}{36}, 1 ;
\end{array}\right]
\end{gathered}
$$

Now write the right hand side of (44) in terms of ordinary Bessel function of order zero, we get the summation theorem (24). Similarly, we can prove (22) and (23).

Now we shall establish (27) from Ramanujan's series $R_{6}$.

$$
\begin{gathered}
\sum_{n=0}^{\infty} \frac{(42 \sqrt{5} n+5 \sqrt{5}+30 n-1)\left(\frac{1}{2}\right)_{n}^{3}}{64^{n}(n !)^{3}}\left(\frac{\sqrt{5}-1}{2}\right)^{8 n}=\frac{32}{\pi} \\
\sum_{n=0}^{\infty} \frac{\{(-1+5 \sqrt{5})+(30+42 \sqrt{5}) n\}\left(\frac{1}{2}\right)_{n}\left(\frac{1}{2}\right)_{n}\left(\frac{1}{2}\right)_{n}}{(1)_{n}(1)_{n} n !}\left\{\frac{(\sqrt{5}-1)^{8}}{2^{14}}\right\}^{n}=\frac{32}{\pi}
\end{gathered}
$$


Now using the Lemma (2) in (45), we get

$$
\begin{aligned}
& \sum_{n=0}^{\infty} \frac{(-1+5 \sqrt{5})\left(\frac{29+47 \sqrt{5}}{30+42 \sqrt{5}}\right)_{n}\left(\frac{1}{2}\right)_{n}\left(\frac{1}{2}\right)_{n}\left(\frac{1}{2}\right)_{n}}{(1)_{n}(1)_{n}\left(\frac{-1+5 \sqrt{5}}{30+42 \sqrt{5}}\right)_{n} n !}\left\{\frac{(-1+\sqrt{5})^{8}}{2^{14}}\right\}^{n}=\frac{32}{\pi} \\
& { }_{4} F_{3}\left[\begin{array}{c}
\left.\frac{1}{2}, \frac{1}{2}, \frac{1}{2}, \frac{29+47 \sqrt{5}}{30+42 \sqrt{5}} ; \frac{(-1+\sqrt{5})^{8}}{2^{14}}\right]=\frac{32}{(-1+5 \sqrt{5}) \pi} \\
1,1, \frac{-1+5 \sqrt{5}}{30+42 \sqrt{5}} ;
\end{array}\right]=\frac{(8+40 \sqrt{5})}{31 \pi} \\
& \text { or }{ }_{4} F_{3}\left[\begin{array}{c}
\left.\frac{1}{2}, \frac{1}{2}, \frac{1}{2}, \frac{(375-8 \sqrt{5})}{330} ; \frac{(47-21 \sqrt{5})}{128}\right]=\frac{(45-8 \sqrt{5})}{330} ;
\end{array}\right]
\end{aligned}
$$

which is the summation theorem (27).

Similarly, in view of the Lemma (2), we can establish the remaining summation theorems (25)-(43), from Ramanujan series $\mathrm{R}_{4}, \mathrm{R}_{5}, \mathrm{R}_{7}, \mathrm{R}_{8}, \mathrm{R}_{9}, \cdots, \mathrm{R}_{20}$, Borwein series $\mathrm{R}_{21}$ and Chudnovsky series $\mathrm{R}_{22}$ respectively.

It may be remarked that the above summations will lead to the excellent value of $\pi$ approximated to various places of decimals, by taking few terms of the Ramanujan series $\mathrm{R}_{4}$ to $\mathrm{R}_{20}$. For example, in $1987, \mathrm{R}$. Wm. Gosper employed the series $\mathrm{R}_{20}$ in calculating 17000000 digits of $\pi$.

Currently, the world record for the most digits of $\pi$ per term in a series of Ramanujan-type for $\frac{1}{\pi}$ is held by Berndt and H. H. Chan (Berndt, B. C, 2001), who derived a series yielding 73 or 74 digits of $\pi$ per term.

\section{Acknowledgement}

The authors are thankful to Prof. R. Y. Denis and Prof. M. A. Pathan for their valuable suggestions during the preparation of the manuscript of this research paper. Second and third authors are also grateful to the referees for their comments for possible improvements in the text of the manuscript, both the authors are highly thankful to the Editor-JMR, for his kindness and coperations for finally publication of this paper.

\section{References}

Berndt, B. C. (1985, 1989, 1991, 1994, 1998). Ramanujan's notebooks. Parts.I-V, Springer-Verlag, New York.

Berndt, B. C. and Chan, H. H. (2001). Eisenstein series and approximations to $\pi$. Illinois J. Math, 45, 75-90.

Berndt, B. C. and Rankin, R. A. (2003). Ramanujan: Essays and Surveys. Hindustan Book Agency (India), New Delhi, Indian Edition.

Borwein, J. M. and Borwein, P. B. (1987). Pi and the AGM. Can. Math. Soc., John Wiley, New york.

Borwein, J. M. and Borwein, P. B. (1987). Ramanujan's rational and algebraic series for $\frac{1}{\pi}$. Indian J. Math, 51, $147-160$.

Borwein, J. M. and Borwein, P. B. (1988). More Ramanujan-type series for $\frac{1}{\pi}$, in Ramanujan Revisited. Academic Press, Boston, 359-374.

Borwein, J. M. and Borwein, P. B. (1993). Class number three Ramanujan-type series for $\frac{1}{\pi}$. J. Comput. Appl. Math, 46, 281-290.

Chudnovsky, D. V. and Chudnovsky, G. V. (1988). Approximations and complex multiplication according to Ramanujan, in Ramanujan Revisited. Academic Press, Boston, 375-472.

Hardy, G. H., Aiyar, P. V. Seshu and Wilson, B. M. (1962). Collected papers of Srinivasa Ramanujan. First published by Cambridge University Press, Cambridge, 1927; Reprinted by Chelsea, New York; Reprinted by the American Mathematical Society, Providence, RI, 2000.

Preston, R. (1992). Profiles: The mountains of $\pi$. The New Yorker (March 2,), 36-67.

Prudnikov, A. P., Brychkov, Yu. A. and Marichev, O. I. (1986). Integrals and series Vol. 3: More special functions. Nauka, Moscow. Translated from the Russian by G. G. Gould, Gordon and Breach Science Publishers, New York, Philadelphia, London, Paris, Montreux, Tokyo, Melbourne, 1990.

Ramanujan, S. (1914). Modular equations and approximations to $\pi$. Quart. J. Math, 45, 350-372.

Ramanujan, S. (1984). Notebooks of Srinivasa Ramanujan. Vol. II, Tata Institute of Fundamental Research, Bombay, 1957; Reprinted by Narosa, New Delhi.

Venkatachala, B. J., Vinay, V. and Yogananda, C. S. (2000). Ramanujan's papers. Prism Books Pvt. Ltd., Bangalore, Mumbai. 